\title{
AN ISOMORPHISM BETWEEN BRANCHED AND GEOMETRIC ROUGH PATHS
}

\author{
HORATIO BOEDIHARDJO AND ILYA CHEVYREV
}

\begin{abstract}
We exhibit an explicit natural isomorphism between spaces of branched and geometric rough paths. This provides a multi-level generalisation of the isomorphism of Lejay-Victoir [LV06 as well as a canonical version of the Itô-Stratonovich correction formula of Hairer-Kelly HK15]. Our construction is elementary and uses the property that the Grossman-Larson algebra is isomorphic to a tensor algebra.

We apply this isomorphism to study signatures of branched rough paths. Namely, we show that the signature of a branched rough path is trivial if and only if the path is tree-like, and construct a non-commutative Fourier transform for probability measures on signatures of branched rough paths. We use the latter to provide sufficient conditions for a random signature to be determined by its expected value, thus giving an answer to the uniqueness moment problem for branched rough paths.
\end{abstract}

\section{Contents}

1. Introduction

2. Preliminaries 4

2.1. Notation 4

2.2. Branched rough paths and the GL algebra 4

2.3. GL algebra as a free algebra 5

3. Geometric П-rough paths 6

3.1. Paths in homogeneous groups 7

4. Branched rough paths are geometric П-rough paths 8

5. Signatures of branched rough paths 11

5.1. Uniqueness of signatures 11

5.2. Fourier transform and moment problem 12

6. Example: Itô-lift of a semi-martingale 16

Appendix A. Symbolic index 18

References

2010 Mathematics Subject Classification. Primary 60H10; Secondary 16T05, 60B15.

Key words and phrases. Branched rough paths, Butcher group, signature, non-commutative Fourier transform. 


\section{INTRODUCTION}

Differential equations of the form

$$
\mathrm{d} Y_{t}=\sum_{i=1}^{d} f_{i}\left(Y_{t}\right) \mathrm{d} X_{t}^{i}, \quad Y_{0}=y \in \mathbb{R}^{e},
$$

where $f_{i}: \mathbb{R}^{e} \rightarrow \mathbb{R}^{e}$ are smooth vector fields and $X=\left(X^{1}, \ldots, X^{d}\right):[0,1] \rightarrow \mathbb{R}^{d}$ is a path, are ubiquitous in control theory and stochastic analysis. A major difficulty in giving meaning to (1.1) in the stochastic setting is that many examples of $X$ (e.g., the sample paths of Brownian motion) are highly irregular, and beyond the reach of Lebesgue-Stieltjes-Young integration theory.

A key observation of Lyons Lyo98 in his introduction of rough paths theory was that equation (1.1) is well-defined in a pathwise sense as long as the driver $X$ is a geometric rough path. One of the successes of rough paths theory has been a separation of probabilistic and deterministic arguments required to solve such differential equations, which has allowed the driver $X$ to be taken well beyond semi-martingale theory (e.g., fractional Brownian motion with Hurst parameter $\left.H>\frac{1}{4}\right)$ as well as given a novel perspective on many classical results in stochastic analysis.

The geometric theory of rough paths is restricted to drivers which respect the usual chain rule of smooth paths. A meaningful generalisation of the theory was provided by Gubinelli's notion of branched rough paths Gub10. Branched rough paths can be seen as an adaptation of Butcher series [But16, which are used in the analysis of non-linear ODEs, to the setting of rough driving paths.

Branched rough paths also fall naturally into Hairer's theory of regularity structures Hai14, in which drivers of singular stochastic PDEs are typically "nongeometric" due to renormalisation procedures required to obtain non-trivial limits. A careful analysis of branched rough paths in this framework was recently carried out in BCFP17, which in turn inspired an algebraic method to renormalise SPDEs in regularity structures $\mathrm{BCCH} 17$. See also $\left[\mathrm{BFG}^{+} 17\right]$ for a recent application of techniques from regularity structures in a setting very close to branched rough paths.

There have been several attempts to reformulate non-geometric as geometric rough paths (the inclusion of geometric into branched/non-geometric rough paths is clear, but a canonical reverse inclusion is not). For $p \in(2,3)$, it was shown in LV06] that the space of non-geometric $p$-rough paths is canonically isomorphic to a space of geometric rough paths over a larger space with mixed $(p, p / 2)$-regularity. This led to a natural (deterministic) Itô formula for rough differential equations. Another approach was taken in [HK15, where the authors showed that to every branched $p$-rough path one can associate a geometric $p$-rough path, also leading to an Itô-type formula. The construction of [HK15, however, is highly non-canonical (relying on the Lyons-Victoir extension theorem [LV07]) and requires a non-trivial transformation of the original branched rough path. It therefore comes short of the satisfactory answer provided in [LV06 for $p \in(2,3)$.

The first main contribution of this article is to exhibit, for any $p \geq 1$, an explicit natural isomorphism between the space of branched $p$-rough paths and a suitable space of geometric rough paths, see Theorem 4.3. This result can be seen as a multi-level generalisation of the result of [LV06], which is important for studying rough signals with 3 -variation (or $\frac{1}{3}$-Hölder) regularity and worse. In turn, 
this isomorphism gives rise to a canonical Itô-Stratonovich correction formula, see Proposition 4.9.

Our construction is elementary and relies on a result of Foissy [Foi02] and Chapoton [Cha10], which was already conjectured by Agrachev-Gamkrelidze [AcG80, that the Grossman-Larson Hopf algebra, in which branched rough paths take values, is isomorphic to a tensor Hopf algebra. Theorem 4.3 follows by combining the result of Foissy-Chapoton with a natural way to measure mixed regularity (we find it convenient for this purpose to work with the notion of ПI-rough paths [Gyu16]).

The second contribution of this article is in the study of signatures of branched rough paths. By reducing to the geometric setting, we show that

(a) (Theorem 5.1) the signature map uniquely determines branched rough paths up to tree-like equivalence,

(b) (Theorem 5.13) there exists a Fourier transform on probability measures on signatures of branched rough paths which uniquely determines measures.

As a consequence of the final point, we give sufficient conditions for the expected value of the signature of a random branched rough path to uniquely determine its law, see Proposition 5.16.

The signature (also known as the Chen-Fliess series) can be seen as the exponential function on path space and arose in Chen's study of the cohomology of loop space [Che57]. It was shown in [HL10 that the signature uniquely determines bounded variation paths up to tree-like equivalence, and this result was recently generalised to all weakly geometric rough paths BGLY16].

In the stochastic setting, it was shown in CL16] that there exists a natural noncommutative Fourier transform (or characteristic function) on random signatures of geometric rough paths, and that, for of a wide class of stochastic processes, the expectation of the signature uniquely determines its law. The terms in the expected signature also arise naturally in stochastic Taylor expansions Aze82, BA89, FV08 and were instrumental in the development of cubature methods on Wiener space [LV04].

The difficulty in proving (a) and (b) for branched rough paths directly is that the techniques developed in BGLY16, CL16 rely on the geometric nature of the rough paths at hand. In particular, as we explain in Section 5.2. one defines a Fourier transform in terms of unitary representations of the signature group, and the study of these representations in CL16 relies crucially on the fact that the signature is a group-like element of a tensor Hopf algebra. That said, the reduction to the geometric setting greatly simplifies (though does not trivialise) both problems.

Remark 1.1. We work in this paper only with rough paths over a finite-dimensional vector space. We note that a framework to address branched rough paths over general Banach spaces has been given in [CW16, and we expect that the isomorphism and uniqueness of signature from Theorems 4.3 and 5.1 carry over to this setting; the construction of a Fourier transform however is more delicate (in [CL16], this was not achieved for geometric rough paths over infinite-dimensional Banach spaces).

The structure of the article is the following. In Section 2 we collect some preliminary material and recall the result of Foissy-Chapoton. In Section 3 we recall and prove several properties of geometric П-rough paths. In Section 4 we make explicit the isomorphism between branched and geometric rough paths, and derive several 
important consequences. In Section 5 we prove the aforementioned results concerning signatures of branched rough paths. We conclude in Section [6 with an explicit example of the isomorphism from Theorem 4.3 applied to lifts of semi-martingales, and show how to verify the conditions of Proposition 5.16 to solve the moment problem.

Acknowledgements. I.C. is funded by a Junior Research Fellowship of St John's College, Oxford. H.B. would like to thank Atul Shekhar for the useful discussions. We would like to thank the anonymous referee for useful suggestions and pointing out the reference $\mathrm{AcG80}$.

\section{Preliminaries}

2.1. Notation. Throughout the paper, we fix $p \geq 1$. For a vector space $V$, we let $T((V))=\prod_{m=0}^{\infty} V^{\otimes m}$ denote the Hopf algebra of formal series in tensors of $V$. By convention, we set $V^{\otimes 0}=\operatorname{span}(\mathbf{1}) \simeq \mathbb{R}$, where $\mathbf{1}$ is the unit of $T((V))$. We denote the product and coproduct on $T((V))$ by $\dot{\otimes}$ and $\Delta$ respectively. Let $T(V)=\bigoplus_{m=0}^{\infty} V^{\otimes m}$ denote the (free) tensor Hopf algebra generated by $V$, which we identify with the subspace of $T((V))$ consisting of finite series.

We denote by $|\cdot|$ a norm on a vector space. Whenever we refer to a norm on a tensor product $V_{1} \otimes \ldots \otimes V_{m}$, where $V_{1}, \ldots, V_{m}$ are normed spaces, we shall always assume this is the projective norm. We then let $V_{1} \hat{\otimes} \ldots \hat{\otimes} V_{m}$ denote its completion. For a locally convex space $V$, we consequently define the algebra $P(V)=\prod_{m=0}^{\infty} V^{\hat{\otimes} m}$. Note that $T((V))$ is a subspace of $P(V)$, and that $T((V))=$ $P(V)$ whenever $V$ is finite dimensional.

2.2. Branched rough paths and the GL algebra. We briefly recall the notion of a branched $p$-rough path over $\mathbb{R}^{d}$. For a graph $\tau$, we let $N_{\tau}$ denote its node set. A labelled rooted tree (henceforth simply tree) is a triple $(\tau, r, \mathcal{L})$, where $\tau$ is a graph which contains no loops, $r \in N_{\tau}$ is a distinguished vertex called the root, and $\mathcal{L}: N_{\tau} \rightarrow\{1, \ldots, d\}$ is a labelling of the nodes of $\tau$. A forest is an (unordered) multiset of trees; by convention, we postulate the empty set, denoted by $\mathbf{1}$, to be a forest. We will denote by $\mathcal{F}$ and $\mathcal{T}$ the set of all forests and trees respectively. Let $\mathcal{B}$ and $\mathcal{H}$ denote the formal linear span over $\mathbb{R}$ of $\mathcal{T}$ and $\mathcal{F}$ respectively. Let $\mathcal{H}^{*}=\left\{\sum_{\sigma \in \mathcal{F}} \lambda_{\sigma} \sigma: \lambda_{\sigma} \in \mathbb{R} \forall \sigma \in \mathcal{F}\right\}$ denote the space of formal series in forests. The space $\mathcal{H}$ contains exactly the series in $\mathcal{H}^{*}$ where all but finitely many terms are zero.

We equip both $\mathcal{H}$ and $\mathcal{H}^{*}$ with the structure of the Grossman-Larson Hopf algebra and denote by $\star$ and $\delta$ the corresponding product and coproduct respectively (note that $\mathbf{1}$ is the unit for $\star$ ). For our purposes, we do not need the precise definitions of $\star$ and $\delta$ and refer the reader to [BCFP17, Sec. 3] and [HK15, Sec. 2] for details.

The Butcher group $\mathcal{G}^{*}$ is defined to be the set of all group-like elements of $\mathcal{H}^{*}$, i.e., $\mathcal{G}^{*}=\left\{g \in \mathcal{H}^{*}: \delta(g)=g \otimes g, g \neq 0\right\}$. One can check that $\mathcal{G}^{*}$ indeed forms a group.

For $\sigma \in \mathcal{F}$, let $|\sigma|$ denote the number of nodes in $\sigma$. For $N \geq 0$, note that the space of series of the form $\sum_{|\sigma|>N} \lambda_{\sigma} \sigma$ is an ideal of $\mathcal{H}^{*}$. We can thus form the corresponding quotient algebra $\mathcal{H}^{N}$ and denote by $\rho^{N}: \mathcal{H}^{*} \rightarrow \mathcal{H}^{N}$ the canonical projection. By construction, the (finite) set $\mathcal{F}^{N}:=\{\sigma \in \mathcal{F}:|\sigma| \leq N\}$ is a basis 
for $\mathcal{H}^{N}$ and we equip $\mathcal{H}^{N}$ with the inner product for which $\mathcal{F}^{N}$ is an orthonormal basis. We define the level- $N$ Butcher group $\mathcal{G}^{N}$ as the image of $\mathcal{G}^{*}$ under $\rho^{N}$.

We also denote by $\mathcal{H}^{(N)}$ the space spanned by $\mathcal{F}^{(N)}:=\{\sigma \in \mathcal{F}:|\sigma|=N\}$ and let $\pi^{N}$ denote the projection $\pi^{N}: \sum_{\sigma} \lambda_{\sigma} \sigma \mapsto \sum_{|\sigma|=N} \lambda_{\sigma} \sigma$, where the series $\sum_{\sigma} \lambda_{\sigma} \sigma$ is understood as an element of $\mathcal{H}^{*}$ or $\mathcal{H}^{M}$ for some $M \geq N$.

Define the simplex $\triangle=\{(s, t): 0 \leq s \leq t \leq 1\}$. A control is a continuous function $\omega: \triangle \rightarrow[0, \infty)$ such that

$$
\omega(s, u)+\omega(u, t) \leq \omega(s, t), \quad \forall(s, u),(u, t) \in \triangle .
$$

Definition 2.1. A branched $p$-rough path is a continuous function $X: \triangle \rightarrow \mathcal{G}^{\lfloor p\rfloor}$ such that

(1) for all $s \leq u \leq t$

$$
X_{s, u} \star X_{u, t}=X_{s, t}
$$

(2) there exists a control $\omega$ such that for all $n=1, \ldots,\lfloor p\rfloor$ and $s \leq t$,

$$
\left|\pi^{n} X_{s, t}\right| \leq \omega(s, t)^{n / p} .
$$

We recall the following extension theorem.

Theorem 2.2 (Gub10]). Let $X: \triangle \rightarrow \mathcal{G}^{\lfloor p\rfloor}$ be a branched $p$-rough path. Then there exists a unique extension $S(X): \triangle \rightarrow \mathcal{H}^{*}$ such that

(1) $\rho^{\lfloor p\rfloor} S(X)=X$;

(2) for all $s \leq u \leq t$,

$$
S(X)_{s, u} \star S(X)_{u, t}=S(X)_{s, t}
$$

(3) for all $n \geq 1$, there exists a control $\omega$ such that $\left|\pi^{n} S(X)_{s, t}\right| \leq \omega(s, t)^{n / p}$.

One can show that the $S(X)_{s, t} \in \mathcal{G}^{*}$ for all $s \leq t$. The element $S(X)_{0,1} \in \mathcal{H}^{*}$ is called the signature of $X$.

2.3. GL algebra as a free algebra. We recall the following result of Foissy Foi02, Sec. 8] and Chapoton Cha10, which will play a central role in the sequel.

Theorem 2.3 ([Foi02, Cha10]). There exists a subspace $B=\operatorname{span}\left(\tau_{1}, \tau_{2}, \ldots\right)$ of $\mathcal{B}$ such that $\mathcal{H}$ is isomorphic as a Hopf algebra to the tensor Hopf algebra $T(B)$.

We note that the subspace $B$ (and evidently choice of basis $\tau_{1}, \tau_{2}, \ldots$ ) is not unique. However, we can and will assume that we have a fixed set of basis elements $\tau_{1}, \tau_{2}, \ldots$ which are in $\mathcal{T}$ and that $\left|\tau_{i}\right| \leq\left|\tau_{j}\right|$ if $i<j$ (cf. [Foi02, p. 106]).

Remark 2.4. This assumption on $\tau_{1}, \tau_{2}, \ldots$ is only for convenience; all our arguments carry through if instead $\tau_{i}=\sum_{j=1}^{n_{i}} c_{i}^{(j)} \tau_{i}^{(j)}$, where every $\tau_{i}^{(j)}$ is an element of $\mathcal{T}$ with $\left|\tau_{i}^{(1)}\right|=\ldots=\left|\tau_{i}^{\left(n_{i}\right)}\right|$.

Due to the grading of $\mathcal{H}$, it follows that every element $\sigma \in \mathcal{H}^{*}$ can be written uniquely as

$$
\sigma=\sum_{m=0}^{\infty} \sum_{r_{1}, \ldots, r_{m}=1}^{\infty} \lambda_{r_{1}, \ldots, r_{m}} \tau_{r_{1}} \star \ldots \star \tau_{r_{m}}
$$

(the term with $m=0$ corresponds to a linear multiple of the empty forest $\mathbf{1}$ ). Note that $\sigma \in \mathcal{H}$ if and only if all but finitely many of the $\lambda$ 's are zero. 


\section{Geometric П-Rough PAths}

In order to describe how every branched rough path is canonically a geometric rough path, we find it most natural to work with a generalisation of geometric p-rough paths which allows one to measure different components with different regularity; the notion of a П-rough path Gyu08, Gyu16, which is a generalisation of $(p, q)$-rough paths [LV06, provides a convenient framework for this purpose.

Remark 3.1. Having different regularities for different components of the "driver" is also naturally achieved in the theory of regularity structures. In fact, the restriction of the algebraic formalism in BHZ16 to the one-dimensional setting (and ignoring the presence of polynomials and derivatives on kernels) yields precisely a "branched" version of $\Pi$-rough paths. The isomorphism between branched and geometric rough paths from Theorem 4.3 then carries over to this setting and reads: every branched $\Pi$-rough path is canonically a geometric $\tilde{\Pi}$-rough path, where $\tilde{\Pi}$ is a tuple of regularities which depends on $\Pi$. In fact, we work with branched $p$-rough paths (i.e., every component of the driver has the same regularity) only for simplicity.

Let $V$ be a finite dimensional normed vector space. We represent different homogeneities of a rough path by decomposing the space $V$ into

$$
V=V^{1} \oplus \ldots \oplus V^{k} .
$$

We define the set of multi-indexes

$$
\mathcal{A}_{k}=\left\{\left(r_{1}, \ldots, r_{m}\right): r_{i} \in\{1, \ldots, k\}, m \geq 0\right\} .
$$

Due to the decomposition (3.1), we may write

$$
T((V))=\prod_{\left(r_{1}, \ldots, r_{m}\right) \in \mathcal{A}_{k}} V^{\otimes R},
$$

where for $R=\left(r_{1}, \ldots, r_{m}\right) \in \mathcal{A}_{k}$ we denote $V^{\otimes R}=V^{r_{1}} \otimes \ldots \otimes V^{r_{m}}$. We let $\pi_{R}: T((V)) \rightarrow V^{\otimes R}$ denote the corresponding projection.

Consider a scaling tuple $\Pi=\left(p_{1}, \ldots, p_{k}\right)$ with $p_{1} \geq \ldots \geq p_{k} \geq 1$. We would like to scale $V^{1}$ by $\frac{1}{p_{1}}, V^{2}$ by $\frac{1}{p_{2}}$, etc. For a multi-index $R=\left(r_{1}, \ldots, r_{m}\right) \in \mathcal{A}_{k}$, we set $n_{j}(R)=\left|\left\{i: r_{i}=j\right\}\right|$ for all $j=1, \ldots, k$, and define the scaling for $V^{\otimes R}$ by

$$
\operatorname{deg}_{\Pi}(R)=\sum_{j=1}^{k} \frac{n_{j}(R)}{p_{j}},
$$

which is the inhomogeneous analogue of the tensor degree.

For $s \geq 0$, define further

$$
\mathcal{A}_{s}^{\Pi}=\left\{R \in \mathcal{A}_{k}: \operatorname{deg}_{\Pi}(R) \leq s\right\} .
$$

Remark 3.2. Note that the set $\mathcal{A}_{s}^{\Pi}$ is finite. In the spirit of Remark 3.1] this fact is directly related to the notion of subcriticality in regularity structures.

Consider the ideal

$$
B_{s}^{\Pi}=\left\{v \in T((V)): \pi_{R}(v)=0 \quad \forall R \in \mathcal{A}_{s}^{\Pi}\right\} .
$$

We define the truncated tensor algebra at degree $s$ as the quotient algebra

$$
T^{(\Pi, s)}(V)=T((V)) / B_{s}^{\Pi} .
$$


Definition 3.3. A П-rough path is a continuous function $X: \triangle \rightarrow T^{(\Pi, 1)}(V)$ such that

(1) for all $s \leq u \leq t$

$$
X_{s, u} \dot{\otimes} X_{u, t}=X_{s, t}
$$

(2) there exists a control $\omega$ such that for all $R \in \mathcal{A}_{1}^{\Pi}$ and $s \leq t$

$$
\left|\pi_{R}\left(X_{s, t}\right)\right| \leq \omega(s, t)^{\operatorname{deg}_{\Pi}(R)} .
$$

The usual extension theorem for $p$-rough paths holds for П-rough paths, namely that a $\Pi$-rough path $X$ has a unique extension $S(X): \triangle \rightarrow T((V))$ which preserves the multiplicative and regularity properties, see Gyu16, Thm. 2.6].

Finally, consider the subgroup $G^{(\Pi, 1)}(V)$ of $T^{(\Pi, 1)}(V)$ defined as the exponential of the Lie subalgebra of $T^{(\Pi, 1)}(V)$ generated by $V$. We say that a $\Pi$-rough path is weakly geometric if it takes values in $G^{(\Pi, 1)}(V)$.

3.1. Paths in homogeneous groups. We describe now how weakly geometric $\Pi$-rough paths can be treated as paths taking values in a homogeneous group. The advantage of this viewpoint is that it gives rise to a homogeneous $p$-variation metric which is convenient to work with in practice (particularly for establishing interpolation estimates). The results here will be helpful later in Section 5.1.

We introduce on $T^{(\Pi, 1)}(V)$ a family of algebra morphisms $\left(\delta_{\lambda}\right)_{\lambda>0}$, called dilation operators, given for any $v \in V^{\otimes R}$ by $\delta_{\lambda}(v)=\lambda^{p_{1} \operatorname{deg}_{\Pi}(R)} v$. Note that the restriction of $\left(\delta_{\lambda}\right)_{\lambda>0}$ to $G^{(\Pi, 1)}(V)$ defines a homogeneous group in the sense of Folland-Stein [FS82] (which is a Carnot group if and only if $p_{1}=\ldots=p_{k}=1$ ).

We equip $G^{(\Pi, 1)}(V)$ with a sub-additive homogeneous norm and corresponding left-invariant metric $d$, see [HS90. Consequently, we define the $p$-variation norm $\|\cdot\|_{p \text {-var }}$ and (homogeneous) $p$-variation metric $d_{p \text {-var }}$ on the space of functions from $[0,1]$ to $G^{(\Pi, 1)}(V)$ (see, e.g., [Che18, Sec. 3.1]).

For maps $X, Y: \triangle \rightarrow T^{(\Pi, 1)}(V)$ and any tuple $\Pi^{\prime}=\left(p_{1}^{\prime}, \ldots, p_{k}^{\prime}\right)$, we define the (inhomogeneous) $\Pi$ '-variation distance

$$
\rho_{\Pi^{\prime}-\operatorname{var}}(\bar{X}, \bar{Y})=\max _{R \in \mathcal{A}_{1}^{\Pi}} \sup _{\mathcal{P}}\left(\sum_{[s, t] \in \mathcal{P}}\left|\pi_{R}\left(\bar{X}_{s, t}-\bar{Y}_{s, t}\right)\right|^{1 / \operatorname{deg}_{\Pi^{\prime}}(R)}\right)^{\operatorname{deg}_{\Pi^{\prime}}(R)},
$$

where $\sup _{\mathcal{P}}$ denotes the supremum over all finite partitions of $[0,1]$ and $\sum_{[s, t] \in \mathcal{P}}$ denotes the sum over adjacent point in $\mathcal{P}$. Note that there is a one-to-one correspondence between multiplicative maps $X: \triangle \rightarrow G^{(\Pi, 1)}(V)$ and paths $\tilde{X}:[0,1] \rightarrow$ $G^{(\Pi, 1)}(V)$ with $\tilde{X}_{0}=\mathbf{1}$, given by $X_{s, t}=\tilde{X}_{s}^{-1} \dot{\otimes} \tilde{X}_{t}$. An analogue of the ball-box estimate [FV10, Prop. 7.49] yields the following lemma.

Lemma 3.4. Let $p \geq 1$. Define $p_{j}^{\prime}=\frac{p}{p_{1}} p_{j}$ and $\Pi^{\prime}=\left(p_{1}^{\prime}, \ldots, p_{k}^{\prime}\right)$. Then the identity map

$$
\left(C^{p-\operatorname{var}}\left([0,1], G^{(\Pi, 1)}(V)\right), d_{p-\operatorname{var}}\right) \leftrightarrows\left(C^{p-\operatorname{var}}\left([0,1], G^{(\Pi, 1)}(V)\right), \rho_{\Pi \Pi^{\prime}-\operatorname{var}}\right)
$$

is Lipschitz on bounded sets in the $\rightarrow$ direction, and Hölder continuous on bounded sets in the $\leftarrow$ direction.

As usual in rough paths theory, the use of this lemma comes from the fact that the Itô-Lyons solution map for RDEs is locally Lipschitz for the metric $\rho_{\Pi \text {-var }}$, while 
interpolation estimates are most easily derived for the metric $d_{p \text {-var }}$. For example, for any $0<p<p^{\prime}$, it holds that (e.g., Che18, Lem. 3.3])

$$
\sup _{t \in[0,1]} d\left(X_{t}^{n}, X_{t}\right) \rightarrow 0 \text { and } \sup _{n}\left\|X^{n}\right\|_{p \text {-var }}<\infty \Rightarrow d_{p^{\prime} \text {-var }}\left(X^{n}, X\right) \rightarrow 0 .
$$

A useful consequence is the following.

Lemma 3.5. Let $X$ be a weakly geometric $\Pi$-rough path. Let $p>p_{1}$ and define $\Pi^{\prime}$ as in Lemma 3.4. Suppose that p is sufficiently close to $p_{1}$ so that $T^{\left(\Pi^{\prime}, 1\right)}(V)=$ $T^{(\Pi, 1)}(V)$. Then $X$ is a geometrid $\Pi^{\prime}$-rough path.

Proof. Observe that there exists $C>0$ such that if $\gamma:[0,1] \rightarrow G^{(\Pi, 1)}(V)$ is the lift of a bounded variation path $\tilde{\gamma}:[0,1] \rightarrow V$ with $|\tilde{\gamma}|_{1 \text {-var }} \leq 1$, then

$$
\|\gamma\|_{\left(p_{1} / p_{k}\right) \text {-var }}^{p_{1} / p_{k}} \leq C|\tilde{\gamma}|_{1-\mathrm{var}} .
$$

By the Rashevsky-Chow theorem, for every $y \in G^{(\Pi, 1)}(V)$ there exists a path $\gamma:[0,1] \rightarrow G^{(\Pi, 1)}(V)$, which is the lift of a Lipschitz path in $V$, such that $\gamma_{0}=\mathbf{1}$ and $\gamma_{1}=y$. By the Arzelà-Ascoli theorem and the lower semi-continuity of $\|\cdot\|_{\left(p_{1} / p_{k}\right) \text {-var }}$, there exists $\gamma$ (not necessarily unique) for which $\|\gamma\|_{\left(p_{1} / p_{k}\right) \text {-var }}$ is minimal amongst all such paths. We call such a $\gamma$ a $\left(p_{1} / p_{k}\right)$-geodesic from 1 to $y$. By (3.3), we see that $\|\gamma\|_{\left(p_{1} / p_{k}\right) \text {-var }} \rightarrow 0$ as $y \rightarrow \mathbf{1}$. It follows that $\|y\|^{\prime}=\|\gamma\|_{\left(p_{1} / p_{k}\right) \text {-var }}$ defines a sub-additive homogeneous norm on $G^{(\Pi, 1)}(V)$, and is therefore equivalent to $\|\cdot\|$.

Let $\mathcal{P}_{n}$ be a sequence of partitions for which the mesh size $\left|\mathcal{P}_{n}\right| \rightarrow 0$. Let $X^{n}$ be a piecewise $\left(p_{1} / p_{k}\right)$-geodesic approximation to $X$ over $\mathcal{P}_{n}$, i.e., for every $[s, t] \in \mathcal{P}_{n}$, the path $[s, t] \rightarrow G^{(\Pi, 1)}(V), u \mapsto X_{s, u}^{n}$, is a (reparametrisation of a) $\left(p_{1} / p_{k}\right)$-geodesic from 1 to $X_{s, t}$. It clearly holds that $X^{n} \rightarrow X$ uniformly. Furthermore, using the equivalence of $\|\cdot\|$ and $\|\cdot\|^{\prime}$, one can apply [Che18, Lem. A.5] to show that $\left\|X^{n}\right\|_{p_{1} \text {-var }} \lesssim\|X\|_{p_{1} \text {-var. }}$. It follows by (3.2) and Lemma 3.4 that $X$ is a geometric $\Pi^{\prime}$-rough path.

\section{Branched rough paths are geometric П-Rough paths}

We proceed to give an explicit isomorphism between branched $p$-rough paths and weakly geometric $\Pi$-rough paths. The key remark throughout this section, which is a direct consequence of Theorem 2.3] is that every element in $\mathcal{H}^{N}$ can be uniquely written as

$$
\sum_{R} \lambda_{R} \tau_{r_{1}} \star \ldots \star \tau_{r_{m}}
$$

where $R=\left(r_{1}, \ldots, r_{m}\right)$ ranges over all multi-indexes for which $\sum_{j=1}^{m}\left|\tau_{r_{j}}\right| \leq N$.

Definition 4.1. Let $k \geq 1$ be the largest integer such that $\left|\tau_{k}\right| \leq p$. Consider the decomposition into one-dimensional subspaces

$$
B_{k}=B^{1} \oplus \ldots \oplus B^{k}
$$

where $B^{j}=\operatorname{span}\left(\tau_{j}\right)$. Define $p_{j}=p /\left|\tau_{j}\right|$ and the tuple

$$
\Pi=\left(p_{1}, p_{2}, \ldots, p_{k}\right) \text {. }
$$

\footnotetext{
${ }^{1} \mathrm{~A} \Pi$-rough path is called geometric if it is in the closure under $\rho_{\Pi \text {-var }}$ of the lifts of $V$-valued bounded variation paths.
} 
We now specialise all the notation from Section 3 by setting $B_{k}=V$ with the corresponding decomposition (4.2). Note that, by construction, every element $\sigma \in T^{(\Pi, 1)}\left(B_{k}\right)$ can be written uniquely as

$$
\sigma=\sum_{\left(r_{1}, \ldots, r_{m}\right) \in \mathcal{A}_{1}^{\Pi}} \lambda_{R} \tau_{r_{1}} \dot{\otimes} \ldots \dot{\otimes} \tau_{r_{m}}
$$

where $\mathcal{A}_{1}^{\Pi}$ is the set of all multi-indexes $R=\left(r_{1}, \ldots, r_{m}\right) \in\{1, \ldots, k\}^{m}, m \geq 0$, for which $\operatorname{deg}_{\Pi}(R)=\sum_{j=1}^{m}\left|\tau_{r_{j}}\right| / p \leq 1$.

Lemma 4.2. There is an algebra isomorphism $\Psi: \mathcal{H}^{\lfloor p\rfloor} \rightarrow T^{(\Pi, 1)}\left(B_{k}\right)$ given, for all $R=\left(r_{1}, \ldots r_{m}\right) \in \mathcal{A}_{1}^{\Pi}$, by

$$
\Psi: \tau_{r_{1}} \star \ldots \star \tau_{r_{m}} \mapsto \tau_{r_{1}} \dot{\otimes} \ldots \dot{\otimes} \tau_{r_{m}} .
$$

Furthermore, there exists $C>0$ such that for all $\tau \in \mathcal{H}^{\lfloor p\rfloor}$ and $n=1, \ldots,\lfloor p\rfloor$, it holds that

$$
\sup _{\operatorname{deg}_{\Pi}(R)=n / p} C^{-1}\left|\pi_{R} \Psi(\tau)\right| \leq\left|\pi^{n} \tau\right| \leq \sup _{\operatorname{deg}_{\Pi}(R)=n / p} C\left|\pi_{R} \Psi(\tau)\right| .
$$

Proof. The existence of $\Psi$ is immediate from the definitions and Theorem 2.3 while (4.3) follows from the equivalence of norms on the finite-dimensional space $\mathcal{H}^{(n)}$.

Recall that the space of maps from $\triangle$ into $\mathcal{H}^{\lfloor p\rfloor}$ and $T^{(\Pi, 1)}\left(B_{n}\right)$ can be equipped respectively with the (inhomogeneous) $p$ - and $\Pi$-variation metrics

$$
\begin{aligned}
\rho_{p-\operatorname{var}}(X, Y) & =\max _{n=1, \ldots,\lfloor p\rfloor} \sup _{\mathcal{P}}\left(\sum_{[s, t] \in \mathcal{P}}\left|\pi^{n}\left(X_{s, t}-Y_{s, t}\right)\right|^{p / n}\right)^{n / p}, \\
\rho_{\Pi-\operatorname{var}}(\bar{X}, \bar{Y}) & =\max _{R \in \mathcal{A}_{1}^{\Pi}} \sup _{\mathcal{P}}\left(\sum_{[s, t] \in \mathcal{P}}\left|\pi_{R}\left(\bar{X}_{s, t}-\bar{Y}_{s, t}\right)\right|^{1 / \operatorname{deg}_{\Pi}(R)}\right)^{\operatorname{deg}_{\Pi}(R)},
\end{aligned}
$$

where, as before, $\sup _{\mathcal{P}}$ denotes the supremum over all finite partitions of $[0,1]$ and $\sum_{[s, t] \in \mathcal{P}}$ denotes the sum over adjacent point in $\mathcal{P}$.

Theorem 4.3. Consider a map $X: \triangle \rightarrow \mathcal{H}^{\lfloor p\rfloor}$. For $\Psi$ as in Lemma 4.2, let $\bar{X}:=\Psi(X): \triangle \rightarrow T^{(\Pi, 1)}\left(B_{k}\right)$. Then

(a) $X$ is multiplicative if and only if $\bar{X}$ is multiplicative,

(b) $X$ takes values in $\mathcal{G}^{\lfloor p\rfloor}$ if and only if $\bar{X}$ takes values in $G^{(\Pi, 1)}\left(B_{k}\right)$,

(c) there exists $C>0$, independent of $X$, such that if $Y: \triangle \rightarrow \mathcal{H}^{\lfloor p\rfloor}$ is another map and $\bar{Y}:=\Psi(Y)$, then

$$
C^{-1} \rho_{\Pi-\operatorname{var}}(\bar{X}, \bar{Y}) \leq \rho_{p \text {-var }}(X, Y) \leq C \rho_{\Pi-\operatorname{var}}(\bar{X}, \bar{Y}) ;
$$

in particular, $X$ is a branched p-rough path if and only if $\bar{X}$ is a weakly geometric $\Pi$-rough path over $B_{k}$ associated with the decomposition (4.2),

(d) suppose that $X$ is a branched p-rough path. Let $S(X): \triangle \rightarrow \mathcal{H}^{*}$ denote the extension of $X$ and $S(\bar{X}): \triangle \rightarrow T\left(\left(B_{k}\right)\right)$ denote the extension of $\bar{X}$. Then $S(X)=\imath S(\bar{X})$, where $\imath: T\left(\left(B_{k}\right)\right) \hookrightarrow P(B)$ is the natural inclusion map, and where we identify $\mathcal{H}^{*}$ with $P(B)$ as in Section 5.2.2.

Proof. Since $\Psi$ is an algebra isomorphism, it holds that $X$ is multiplicative if and only $\bar{X}$ is, which proves (a). Likewise, $\log (X)$ takes values in the Lie subalgebra of $\mathcal{H}^{\lfloor p\rfloor}$ generated by the trees $\tau_{1}, \ldots, \tau_{k}$ if and only if $\log (\bar{X})$ takes values in the 
Lie subalgebra of $T^{(\Pi, 1)}\left(B_{k}\right)$ generated by $\tau_{1}, \ldots, \tau_{k}$, which proves $(\mathrm{b})$. Next, the bound (4.4) is an immediate consequence of (4.3) and the definition of $\rho_{p \text {-var }}$ and $\rho_{\Pi-\mathrm{var}}$, which proves $(\mathrm{c})$.

To prove (d) consider $N \geq\lfloor p\rfloor$ and $Z=\rho^{N} \imath S(\bar{X}): \triangle \rightarrow \mathcal{H}^{N}$. It is immediate that $\rho^{\lfloor p\rfloor} Z=X$, and, since $\imath$ and $\rho^{N}$ are algebra morphisms, that $Z$ is a multiplicative map. Furthermore, using that $S(\bar{X})$ has finite $\Pi$-variation Gyu16. Thm. 2.6], it readily follows from an analogous bound to 4.3 that $Z$ has finite $p$-variation. By uniqueness of the branched rough path lift, it follows that $Z=\rho^{N} S(X)$, and thus $S(X)=\imath S(\bar{X})$ as desired.

Remark 4.4. The reader may wonder how canonical our interpretation of $X$ as a geometric П-rough path is, given that the space $B$ and the decomposition (4.2) depend on the choice of basis $\tau_{1}, \tau_{2}, \ldots$ It is easy to see, however, that a different choice of $\tau_{1}, \tau_{2}, \ldots$ will lead to canonically isomorphic objects (provided $\tau_{i}$ are chosen in accordance with Remark 2.4).

Remark 4.5. The isomorphism between geometric and non-geometric rough paths shown in LV06 is precisely the case $p \in(2,3)$ of Theorem 4.3

Remark 4.6. In [HK15], for any branched p-rough path, the authors employ the Lyons-Victoir extension theorem [LV07] to construct a geometric $p$-rough path taking values in the tensor algebra over $\mathcal{B}^{\lfloor p\rfloor}=\operatorname{span}(\tau \in \mathcal{T}:|\tau| \leq\lfloor p\rfloor)$. In contrast, the isomorphism in Theorem 4.3 does not "extend" the branched rough path in any way and instead treats its target space as a different algebraic structure. The explicit nature of this isomorphism will be particularly important in our study of the signature in Section 5 .

We now present two consequences of Theorem 4.3 For the remainder of this section, suppose that $X: \triangle \rightarrow \mathcal{G}^{\lfloor p\rfloor}$ is a branched $p$-rough path and that $\bar{X}=$ $\Psi(X): \triangle \rightarrow T^{(B, 1)}\left(B_{k}\right)$. As before, denote by $S(X): \triangle \rightarrow \mathcal{H}^{*}$ the extension of $X$.

First, it follows from Theorem 4.3 that the level- $N$ lift of a branched $p$-rough path is the solution of a linear differential equation driven by a geometric $\Pi$-rough path.

Corollary 4.7. Let $N \geq\lfloor p\rfloor$ and let $Y=\rho^{N} S(X)_{0, \cdot}:[0,1] \rightarrow \mathcal{G}^{N}$. Then $Y$ is the solution of the linear $R D E$

$$
\mathrm{d} Y=f(Y) \mathrm{d} \bar{X}
$$

where $f=\left(f_{1}, \ldots, f_{k}\right)$ are the (left-invariant) vector fields on $\mathcal{H}^{N}$ given by rightmultiplication by $\left(\tau_{1}, \ldots, \tau_{k}\right)$ respectively.

The interest in the above corollary stems from the fact that in general one is not able to describe $Y$ as the solution of a linear RDE driven by the original branched rough path $X$, cf. [BCFP17, Rem. 34].

Second, we show an Itô-type formula that any RDE driven by $X$ coincides in a natural way with an RDE driven by $\bar{X}$. For the remainder of this section, consider bounded smooth vector fields $f=\left(f_{1}, \ldots, f_{d}\right)$ on $\mathbb{R}^{e}$ with bounded derivatives of all orders 2

\footnotetext{
${ }^{2}$ The regularity assumptions on $f$ can be significantly weakened, see the sharp version of the universal limit theorem for geometric П-rough paths Gyu16 Thm. 4.3]; we restrict to smooth vector fields only for simplicity.
} 
Recall the pre-Lie product $\curvearrowright: \mathcal{B} \times \mathcal{B} \rightarrow \mathcal{B}$ defined by $\tau \curvearrowright \sigma=\pi_{\mathcal{B}}(\tau \star \sigma)$, where $\pi_{\mathcal{B}}: \mathcal{H} \rightarrow \mathcal{B}$ is the projection onto $\mathcal{B}$. Explicitly, $\tau \curvearrowright \sigma=\sum_{\bar{\tau}} n(\tau, \sigma, \bar{\tau}) \bar{\tau}$, where the sum is over all trees $\bar{\tau} \in \mathcal{T}$ and $n(\tau, \sigma, \bar{\tau})$ is the number of single admissible cuts of $\bar{\tau}$ for which the branch is $\tau$ and the trunk is $\sigma$. Recall also that the space of vector fields $C^{\infty}\left(\mathbb{R}^{e}, \mathbb{R}^{e}\right)$ can be equipped with a pre-Lie product defined by $f \triangleleft g=\sum_{i=1}^{e} f^{i} \partial_{i} g$. By a result of Chapoton-Livernet [CL01, we can identify $\mathcal{B}$ with the free pre-Lie algebra over $\mathbb{R}^{d}$, and thus there exists a unique pre-Lie algebra morphism $\mathcal{B} \rightarrow C^{\infty}\left(\mathbb{R}^{e}, \mathbb{R}^{e}\right), \tau \mapsto f_{\tau}$, for which $f_{\bullet}=f_{i}$ for all $i=1, \ldots, d$.

Remark 4.8. Every tree $\tau \in \mathcal{T}$ can be written as $\tau=\left[\sigma_{1} \ldots \sigma_{n}\right]_{i}$ (which is unique up to permutation of the $\sigma_{j}$ ), by which we mean that $\tau$ is formed by attaching the trees $\sigma_{1}, \ldots, \sigma_{n} \in \mathcal{T}$ to a root with label $i \in\{1, \ldots, d\}$ (if $n=0$, we have $\tau=\bullet_{i}$ ). For $\tau=\left[\sigma_{1} \ldots \sigma_{n}\right]_{i} \in \mathcal{T}$, the vector field $f_{\tau}$ admits the inductive form

$$
f_{\tau}=c_{\tau}\left(D^{n} f_{i}\right)\left(f_{\sigma_{1}}, \ldots, f_{\sigma_{n}}\right),
$$

where $c_{\tau}$ is a combinatorial factor expressible in terms of the symmetries of $\tau$.

Proposition 4.9. Define the vector fields $\bar{f}=\left(\bar{f}_{1}, \ldots, \bar{f}_{k}\right)=\left(f_{\tau_{1}}, \ldots, f_{\tau_{k}}\right)$ on $\mathbb{R}^{e}$. Then the unique solutions to the (branched) $R D E \mathrm{~d} Y=f(Y) \mathrm{d} X$ and the (geometric) $R D E \mathrm{~d} \bar{Y}=\bar{f}(\bar{Y}) \mathrm{d} \bar{X}$ coincide.

Proof. Recall that $Y$ and $\bar{Y}$ are characterised by the Euler estimates

$$
Y_{s, t}=\sum_{|\tau| \leq\lfloor p\rfloor} f_{\pi_{B} X_{s, t}}\left(Y_{s}\right)+o(\omega(s, t))
$$

(where we treat $X_{s, t}$ as an element of $\mathcal{H}$ by the embedding $\mathcal{H}^{\lfloor p\rfloor} \hookrightarrow \mathcal{H}$ ) and

$$
\bar{Y}_{s, t}=\sum_{\left(r_{1}, \ldots, r_{m}\right) \in \mathcal{A}_{1}^{\Pi}} \bar{f}_{r_{1}} \ldots \bar{f}_{r_{m}} I\left(\bar{Y}_{s}\right)\left\langle\bar{X}_{s, t}, \tau_{r_{1}} \dot{\otimes} \ldots \dot{\otimes} \tau_{r_{m}}\right\rangle+o(\omega(s, t)),
$$

where $\omega$ is a control on the $p$-variation of $X$ and $\bar{X}$ (for the former, see HK15, Prop. 3.8] ${ }^{3}$ for the latter, see [FV10, Cor. 10.15]). To conclude that $Y$ and $\bar{Y}$ coincide, it remains to observe that

$$
\sum_{\left(r_{1}, \ldots, r_{m}\right) \in \mathcal{A}_{1}^{\Pi}} \bar{f}_{r_{1}} \ldots \bar{f}_{r_{m}} I(y)\left\langle\bar{X}_{s, t}, \tau_{r_{1}} \dot{\otimes} \ldots \dot{\otimes} \tau_{r_{m}}\right\rangle=\bar{f}_{X_{s, t}} I(y)=f_{\pi_{\mathcal{B}} X_{s, t}}(y),
$$

where $\bar{f}_{\tau}$ denotes the image of $\tau \in \mathcal{H}$ under the unique algebra morphism $\mathcal{H} \rightarrow$ $\mathcal{O}\left(\mathbb{R}^{e}\right)$ which maps $\tau_{r} \mapsto f_{\tau_{r}}$ for $r=1,2, \ldots$, where $\mathcal{O}\left(\mathbb{R}^{e}\right)$ is the algebra of differential operators on $\mathbb{R}^{e}$ (this algebra morphism exists due to Theorem 2.3).

\section{Signatures of BRAnChed ROUgh Paths}

5.1. Uniqueness of signatures. We now apply the identification of branched $p$ rough paths and geometric П-rough paths to prove the following characterisation of branched rough paths with trivial signature.

For a topological space $\mathcal{S}$, recall that a continuous path $X:[0,1] \rightarrow \mathcal{S}$ is called tree-like if there exists an $\mathbb{R}$-tree $\mathfrak{T}$, a continuous function $\phi:[0,1] \rightarrow \mathfrak{T}$, and a map $\psi: \mathfrak{T} \rightarrow \mathcal{S}$ such that $\phi(0)=\phi(1)$ and $X=\psi \circ \phi$.

Theorem 5.1. Let $X: \triangle \mapsto \mathcal{G}^{\lfloor p\rfloor}$ be a branched p-rough path. Then $S(X)_{0,1}=1$ if and only if $X_{0, .}$ is tree-like.

\footnotetext{
${ }^{3}$ Note though that the factors $c_{\tau}$ in 4.5 are missing from the definition of $f_{\tau}$ in HK15
} 
We will first prove that a tree-like branched rough path has trivial signature, which, by Theorem 4.3, is equivalent to showing that a weakly geometric treelike $\Pi$-rough path has trivial signature. The proof is effectively identical to that of geometric rough paths case [BGLY16, Thm. 1.1], but we find it necessary to emphasise several details.

Lemma 5.2. Let notation be as in Section 3. Let $X: \triangle \rightarrow T^{(\Pi, 1)}(V)$ be a weakly geometric П-rough path, and define

$$
(\overleftarrow{X})_{s, t}=X_{1-t, 1-s}^{-1}
$$

Then for all $s \leq t$,

$$
S(\overleftarrow{X})_{1-t, 1-s} \dot{\otimes} S(X)_{s, t}=\mathbf{1}
$$

Proof. The claim is clearly true if $X$ has bounded variation (as a path in $V$ ) and the conclusion follows by density and Lemma 3.5

Remark 5.3. While we state Lemma 5.2 only for weakly geometric П-rough paths, a direct (albeit more involved) argument shows that the same result holds true for any $\Pi$-rough path (not necessarily weakly geometric) and Banach space $V$.

Proposition 5.4. Let $X$ be a weakly geometric П-rough path for which $X_{0, \text {. is }}$ tree-like. Then $S(X)_{0,1}=\mathbf{1}$.

Proof. The proof in BGLY16, Sec. 3] for weakly geometric $p$-rough paths carries over to our present setting mutatis mutandis. Indeed,

- the "central case" [BGLY16, Lem. 3.1] follows in the identical way by applying Lemma 5.2, and

- the proof in BGLY16, Sec. 3.3] follows in the identical way once we use Lemma 3.4, the interpolation result (3.2), and that the signature map is a continuous function in the metric $\rho_{\Pi{ }^{\prime}-\mathrm{var}}$ Gyu08, Lem. 2.1.2].

Proof of Theorem 5.1. Denote as before $\bar{X}=\Psi(X)$. Note that $\bar{X}$ is tree-like if and only if $X$ is. Furthermore, by part (c) of Theorem $4.3, \bar{X}$ is a weakly geometric $\Pi$-rough path and, by part $(\mathrm{d})$ of Theorem 4.3 , it holds that $S(X)_{0,1}=1$ if and only if $S(\bar{X})_{0,1}=\mathbf{1}$. The "if" direction now follows from Proposition 5.4, while the "only if" direction follows from the main result of BGLY16 (one simply notes that every weakly geometric П-rough path lifts canonically to a weakly geometric $p_{1}$-rough path over $B_{k}$ ).

5.2. Fourier transform and moment problem. We now discuss the Fourier transform (or characteristic function) and moment problem for signatures of branched rough paths. The results here employ Theorem 2.3 to identify $\mathcal{H}$ with the tensor alegbra $T(B)$, which allows us to extend the methods of [CL16].

5.2.1. Universal locally $m$-convex algebra over $B$. We begin by constructing a certain universal topological algebra over $B$.

Remark 5.5. Throughout this subsection, we use no special structure of $B$ and note that it may be replaced by $c_{00}$, the vector space of $\mathbb{R}$-valued sequences which are eventually zero. 
We equip $B$ with the product topology given by the sequence of semi-norms $\left(\gamma_{k}\right)_{k \geq 1}$

$$
\gamma_{k}\left(\sum_{r} \lambda_{r} \tau_{r}\right)=\sum_{r=1}^{k}\left|\lambda_{r}\right|
$$

Let $E_{a}(B)$ denote the topological algebra formed by equipping the tensor algebra $T(B)$ with the corresponding universal locally $m$-convex topology, see CL16. Sec. 2]. Explicitly, a fundamental family of sub-multiplicative semi-norms on $E_{a}(B)$ is given by $\left\{\exp \left(K \gamma_{k}\right)\right\}_{k \geq 1, K>0}$, where

$$
\exp \left(K \gamma_{k}\right)=\sum_{n=0}^{\infty} K^{n} \gamma_{k}^{\otimes n}
$$

and

$$
\gamma_{k}^{\otimes n}\left(\sum_{m=0}^{\infty} \sum_{r_{1}, \ldots, r_{m}=1}^{\infty} \lambda_{r_{1}, \ldots, r_{m}} \tau_{r_{1}} \dot{\otimes} \ldots \dot{\otimes} \tau_{r_{m}}\right)=\sum_{r_{1}, \ldots, r_{n}=1}^{k}\left|\lambda_{r_{1}, \ldots, r_{n}}\right|
$$

(as usual, the term with $m=0$ on the LHS corresponds to a linear multiple of $\left.1 \in B^{\otimes 0}\right)$. Note that all sums above are finite.

For $m \geq 1$, the (complete) locally convex space $B^{\hat{\otimes} m}$ can be identified with the space of formal series

$$
\sum_{r_{1}, \ldots, r_{m}=1}^{\infty} \lambda_{r_{1}, \ldots, r_{m}} \tau_{r_{1}} \dot{\otimes} \ldots \dot{\otimes} \tau_{r_{m}}
$$

and so $P(B)=\prod_{m \geq 0} B^{\hat{\otimes} m}$ can be identified with the space of formal series

$$
\sum_{m=0}^{\infty} \sum_{r_{1}, \ldots, r_{m}=1}^{\infty} \lambda_{r_{1}, \ldots, r_{m}} \tau_{r_{1}} \dot{\otimes} \ldots \dot{\otimes} \tau_{r_{m}} .
$$

Let $E(B)$ denote the completion of $E_{a}(B)$. The following lemma is immediate from the above discussion (and is a special case of [CL16, Cor. 2.5]).

Lemma 5.6. The space $E(B)$ can be identified with the subspace of $P(B)$ consisting of series

$$
\sigma=\sum_{m=0}^{\infty} \sum_{r_{1}, \ldots, r_{m}=1}^{\infty} \lambda_{r_{1}, \ldots, r_{m}} \tau_{r_{1}} \dot{\otimes} \ldots \dot{\otimes} \tau_{r_{m}}
$$

such that for every $k \geq 1$ and $K>0$

$$
\exp \left(K \gamma_{k}\right)(\sigma)=\sum_{m=0}^{\infty} \sum_{r_{1}, \ldots, r_{m}=1}^{k} K^{m}\left|\lambda_{r_{1}, \ldots, r_{m}}\right|<\infty .
$$

We note that $E(B)$ is metrizable and separable since $B$ is (see CL16, Cor. 2.4]). Moreover, recall from [CL16, Sec. 3] that the coproduct $\Delta: E_{a}(B) \rightarrow E_{a}(B) \otimes$ $E_{a}(B)$, defined as usual by $\Delta(\tau)=\tau \otimes \mathbf{1}+\mathbf{1} \otimes \tau$ for all $\tau \in B$ and extended uniquely as an algebra morphism, is continuous, and so extends to the completions $\Delta: E(B) \rightarrow E(B) \hat{\otimes} E(B)$.

Definition 5.7. Let $G=\{g \in E(B): \Delta g=g \otimes g, g \neq 0\}$ denote the set of group-like elements of $E(B)$.

Note that $G$ is a Polish space and a topological group. 
5.2.2. Topology on $\mathcal{H}$. By Theorem 2.3, there is a Hopf algebra isomorphism $\mathcal{H} \simeq$ $E_{a}(B)$, and we henceforth equip $\mathcal{H}$ with the locally $m$-convex topology induced by this isomorphism. We let $\hat{\mathcal{H}}$ denote the completion of $\mathcal{H}$ and note that the previous isomorphism extends to $\hat{\mathcal{H}} \simeq E(B)$ as locally $m$-convex algebras. We continue to use $\exp \left(K \gamma_{k}\right)$ for the semi-norms on $\mathcal{H}$ induced by this isomorphism. That is, a fundamental family of sub-multiplicative semi-norms on $\mathcal{H}$ is given by $\left\{\exp \left(K \gamma_{k}\right)\right\}_{k \geq 1, K>0}$, where

$$
\exp \left(K \gamma_{k}\right)=\sum_{n=0}^{\infty} K^{n} \gamma_{k}^{\otimes n}
$$

and

$$
\gamma_{k}^{\otimes n}\left(\sum_{m=0}^{\infty} \sum_{r_{1}, \ldots, r_{m}=1}^{\infty} \lambda_{r_{1}, \ldots, r_{m}} \tau_{r_{1}} \star \ldots \star \tau_{r_{m}}\right)=\sum_{r_{1}, \ldots, r_{n}=1}^{k}\left|\lambda_{r_{1}, \ldots, r_{n}}\right| .
$$

Note that the above sums are all finite. Remark that while the semi-norms $\exp \left(K \gamma_{k}\right)$ on $\mathcal{H}$ depend on the choice of basis of $B$, the locally $m$-convex topology on $\mathcal{H}$ does not.

Since every element in $\mathcal{H}^{*}$ admits a unique representation as (2.1), we can identify $\mathcal{H}^{*}$ with $P(B)=\prod_{m \geq 0} B^{\hat{\otimes} m}$. In particular, by Lemma 5.6, we can identify $\hat{\mathcal{H}}$ with a subspace of $\mathcal{H}^{*}$ consisting of formal series with a suitable decay condition.

Recall the set of group-like elements $\mathcal{G}^{*}=\left\{g \in \mathcal{H}^{*}: \delta g=g \otimes g, g \neq 0\right\}$.

Definition 5.8. Let $\mathcal{G}=\mathcal{G}^{*} \cap \hat{\mathcal{H}}$ denote the set of group-like elements in $\hat{\mathcal{H}}$. Furthermore, for $k \geq 1$, let $\mathcal{G}_{k}=\mathcal{G} \cap P\left(B_{k}\right)$ denote the group-like elements in $\hat{\mathcal{H}}$ generated by $\tau_{1}, \ldots, \tau_{k}$, where we canonically identify $P\left(B_{k}\right)$ with a subalgebra of $\mathcal{H}^{*}$.

Note that $\mathcal{G}$ is precisely the image of $G$ under the isomorphism $E(B) \simeq \hat{\mathcal{H}}$. Equivalently, $\mathcal{G}$ is the subset of all $g \in \mathcal{G}^{*}$ for which $\exp \left(K \gamma_{n}\right)(g)<\infty$ for all $n \geq 1$ and $K>0$.

Let $k$ be as in Definition 4.1. Then $\mathcal{G}_{k}$ consists of all $g \in \mathcal{G}$ whose unique series representation as (2.1) contains no terms $\tau$ for which $|\tau|>\lfloor p\rfloor$, i.e.,

$$
g=\sum_{m=0}^{\infty} \sum_{r_{1}, \ldots, r_{m}=1}^{k} \lambda_{r_{1}, \ldots, r_{m}} \tau_{r_{1}} \star \ldots \star \tau_{r_{m}} .
$$

An immediate consequence of Theorem 4.3 part (d) along with the factorial decay of geometric П-rough paths Gyu16. Thm. 2.6], is that the signature of a branched $p$-rough path takes values in $\mathcal{G}_{k}$. More precisely, we have the following corollary.

Corollary 5.9. Let $p \geq 1$ and $X$ a branched $p$-rough path. For every $(s, t) \in \triangle$, it holds that $S(X)_{s, t}$ can be uniquely written as

$$
S(X)_{s, t}=\sum_{m=0}^{\infty} \sum_{r_{1}, \ldots, r_{m}=1}^{k} \lambda_{r_{1}, \ldots, r_{m}}^{s, t} \tau_{r_{1}} \star \ldots \star \tau_{r_{m}},
$$

where, for all $K>0$,

$$
\sum_{m=0}^{\infty} \sum_{r_{1}, \ldots, r_{m}=1}^{k} K^{m}\left|\lambda_{r_{1}, \ldots, r_{m}}^{s, t}\right|<\infty
$$


Remark 5.10. We note that it is difficult to use Theorem 4.3 to obtain information about the individual quantities $\left\langle S(X)_{s, t}, \tau\right\rangle$ for $\tau \in \mathcal{F}$. Nonetheless, using an independent method, it was shown in Boe15. Thm. 4] that $\left|\left\langle S(X)_{0,1}, \tau\right\rangle\right| \lesssim c^{|\tau|} \tau !^{-1 / p}$ uniformly over $\tau \in \mathcal{F}$, where $\tau$ ! denotes the tree factorial.

5.2.3. Non-commutative Fourier transform. For the remainder of the section, we identify $\mathcal{H}$ with $E_{a}(B)$ and $\hat{\mathcal{H}}$ with $E(B)$ as topological algebras; we also identify $\mathcal{G}$ with $G$ as topological (Polish) groups.

Our main motivation for the construction of $\hat{\mathcal{H}}$ as the complete universal locally $m$-convex algebra over $B$ is that one can readily characterise the continuous representations of $\hat{\mathcal{H}}$. Indeed, for any Banach algebra $A$, there is a natural bijection between continuous linear maps $M: B \rightarrow A$ and continuous algebra morphisms $M: \hat{\mathcal{H}} \rightarrow A$. Furthermore, for any normed space $V$, the set of continuous linear maps $M: B \rightarrow V$ has a straightforward characterisation: a linear map $M: B \rightarrow V$ is continuous if and only if there exists $N \geq 1$ such that $M\left(\tau_{j}\right)=0$ for all $j \geq N$.

For a complex finite-dimensional Hilbert space $H$, let $\mathbf{L}(H)$ denote the algebra of linear operators on $H$, and $\mathfrak{u}(H)$ the Lie subalgebra of $\mathbf{L}(H)$ consisting of antiHermitian operators on $H$.

Definition 5.11. Let $\mathcal{A}$ denote the family of all continuous finite-dimensional algebra representations $M: \hat{\mathcal{H}} \rightarrow \mathbf{L}\left(H_{M}\right)$ which arise from extensions of continuous linear maps $M: B \rightarrow \mathfrak{u}\left(H_{M}\right)$, where $H_{M}$ ranges over all finite-dimensional complex Hilbert space. We define the corresponding set of matrix coefficients by

$$
\mathcal{C}=\left\{\sigma \mapsto\langle M(\sigma) u, v\rangle_{H_{M}}: M \in \mathcal{A}, u, v \in H_{M}\right\} .
$$

Note that $\mathcal{C}$ consists of $\mathbb{C}$-valued continuous linear functionals on $\hat{\mathcal{H}}$. It readily follows that the restriction of any $M \in \mathcal{A}$ to $\mathcal{G}$ is a continuous group morphism into the compact group of unitary operators on $H_{M}$ (see [CL16, Sec. 4]). Moreover, by considering adjoint and tensor product representations, one can easily show that $\left.\mathcal{C}\right|_{\mathcal{G}}$ is closed under multiplication and conjugation, and is therefore a $*$-subalgebra of $C_{b}(\mathcal{G}, \mathbb{C})$. To summarise, we have the following lemma.

Lemma 5.12. The set $\left.\mathcal{C}\right|_{\mathcal{G}}$ is a subspace of $C_{b}(\mathcal{G}, \mathbb{C})$ which is closed under multiplication and conjugation.

In order to apply a Stone-Weierstrass argument, the final and deeper point left to observe is that $\mathcal{C}$ separates the points of $\hat{\mathcal{H}}$. Remark that if $B$ were finitedimensional, this would follow directly from CL16, Thm. 4.8]. Although $B$ is infinite dimensional, it holds that every element in $B^{\hat{\otimes} m}$ admits the form (5.1). We can thus apply the result of Giambruno-Valenti [GV95, Thm. 6] on polynomial identities of symplectic Lie algebras to conclude that, for every non-zero $\tau \in B^{\hat{\otimes} m}$, there exists $M \in \mathcal{A}$ such that $M(\tau) \neq 0$. It follows from the identification of $\hat{\mathcal{H}}$ with a space of formal series from Lemma 5.6 that for every $\tau \in \hat{\mathcal{H}}$,

$$
M(\tau)=0, \forall M \in \mathcal{A} \Leftrightarrow \tau=0
$$

(cf. proof of [CL16, Thm. 4.8]). Using a Stone-Weierstrass argument, we obtain the following generalisation of [CL16, Cor. 4.12].

Theorem 5.13. Let $\mu, \nu$ be two Borel probability measures on $\mathcal{G}$. It holds that $\mu=\nu$ if and only if $\mu(f)=\nu(f)$ for all $f \in \mathcal{C}$, or equivalently, $\mu(M)=\nu(M)$ for all $M \in \mathcal{A}$. 
Theorem 5.13 naturally suggests the following definition.

Definition 5.14 (Fourier transform). The abstract non-commutative Fourier transform of a probability measure $\mu$ on $\mathcal{G}$ is the map $\hat{\mu}: \mathcal{C} \rightarrow \mathbb{C}, \hat{\mu}: f \mapsto \mu(f)$, or equivalently, the map $\hat{\mu}: M \mapsto \mu(M)$, where $M \in \mathcal{A}$.

5.2.4. Moment problem. With the Fourier transform in hand, we are ready to address the moment-problem. Let $\mathbf{X}$ be a $\mathcal{G}$-valued random variable for which $\langle\mathbf{X}, \sigma\rangle$ is integrable for every $\sigma \in \mathcal{F}$ (e.g., $\mathbf{X}=S(X)_{0,1}$ for a random branched $p$-rough path with controlled moments).

Definition 5.15. We call the element of $\mathcal{H}^{*}$

$$
\operatorname{ESig}(\mathbf{X})=\sum_{\sigma \in \mathcal{F}} \mathbb{E}[\langle\mathbf{X}, \sigma\rangle] \sigma
$$

the expected signature of $\mathbf{X}$.

Combining Theorem 5.13 with [CL16, Prop. 3.2], we arrive at the following solution to the moment problem.

Proposition 5.16 (Moment problem). Suppose $\mathbf{X}$ is a $\mathcal{G}$-valued random variable such that $\operatorname{ESig}(\mathbf{X})$ exists and lies in $\hat{\mathcal{H}}$, i.e.,

$$
\exp \left(K \gamma_{k}\right)(\operatorname{ESig}(\mathbf{X}))<\infty, \quad \forall K>0, k \geq 1,
$$

where $\exp \left(K \gamma_{k}\right)$ is given by (5.2). If $\mathbf{Y}$ is another $\mathcal{G}$-valued random variable such that $\operatorname{ESig}(\mathbf{X})=\operatorname{ESig}(\mathbf{Y})$, then $\mathbf{X}$ and $\mathbf{Y}$ and equal in law.

Remark 5.17. The reader may wonder the extent to which it is possible to control the quantities $\exp \left(K \gamma_{k}\right)(\operatorname{ESig}(\mathbf{X}))$ given that it requires us to derive the form

$$
\operatorname{ESig}(\mathbf{X})=\sum_{m=0}^{\infty} \sum_{r_{1}, \ldots, r_{m}}^{\infty} \lambda_{r_{1}, \ldots, r_{m}} \tau_{r_{1}} \star \ldots \star \tau_{r_{m}},
$$

and there does not seem to be an easy way to determine the values $\lambda_{r_{1}, \ldots, r_{m}}$ from the expression (5.3).

In the case $\mathbf{X}=S(X)_{0,1}$ for a branched $p$-rough path $X$, however, we point out that the form (5.5) can in fact arise more naturally than (5.3) once we identify $X$ with a geometric rough path. Furthermore, checking the bound (5.4) can also become simpler due to the fact that

(a) by Corollary [5.9] the expression [5.5) has no terms $\tau_{r}$ with $\left|\tau_{r}\right|>p$, and thus it suffices to check (5.4) only for $k$ as in Definition 4.1.

(b) a number of methods are available to bound the expected signature of geometric rough paths, one of the most applicable being better-than-exponential tails on the local $p$-variation [CLL13, see [CL16, Cor. 6.5].

\section{EXAMPLE: Itô-LIFT OF A SEMI-MARTINGALE}

Suppose $p \in(2,3)$ and $\left(X^{i}\right)_{i=1}^{d}:[0,1] \rightarrow \mathbb{R}^{d}$ is a semi-martingale. Let $X: \triangle \rightarrow$ $\mathcal{G}^{2}$ be the corresponding Itô branched $p$-rough path defined by

$$
X_{s, t}=\mathbf{1}+\sum_{i=1}^{d} \bullet_{i} X_{s, t}^{i}+\sum_{1 \leq i \leq j \leq d} \bullet_{i} \bullet_{j} X_{s, t}^{i} X_{s, t}^{j}+\sum_{i, j=1}^{d}\left[\bullet_{i}\right]_{j} \int_{s}^{t} X_{s, u}^{i} \mathrm{~d} X_{u}^{j}
$$


where $\left[\bullet_{i}\right]_{j}$ is defined in Remark 4.8 and the integral is defined in the sense of Itô. Recall the subspace of trees $B_{k}$ from Definition 4.1. Using the basic identity

$$
\bullet_{i} \star \bullet_{j}= \begin{cases}\bullet_{i} \bullet_{j}+\left[\bullet_{i}\right]_{j} & \text { if } i \neq j, \\ 2 \bullet_{i} \bullet_{i}+\left[\bullet_{i}\right]_{i} & \text { if } i=j,\end{cases}
$$

it is easy to check in this case that $k=\frac{d(d+3)}{2}$ and a suitable basis for $B_{k}$ is

$$
\left\{\bullet_{i}: 1 \leq i \leq d\right\} \cup\left\{\left[\bullet_{i}\right]_{j}: 1 \leq i \leq j \leq d\right\} .
$$

Furthermore, using the identities

$$
\begin{aligned}
& \int_{s}^{t} X_{s, u}^{i} \mathrm{~d} X_{u}^{j}=\int_{s}^{t} X_{s, u}^{i} \circ \mathrm{d} X_{u}^{j}-\frac{1}{2}\left[X^{i}, X^{j}\right]_{s, t}, \\
& X_{s, t}^{i} X_{s, t}^{j}=\int_{s}^{t} X_{s, u}^{i} \circ \mathrm{d} X_{u}^{j}+\int_{s}^{t} X_{s, u}^{j} \circ \mathrm{d} X_{u}^{i},
\end{aligned}
$$

where od denotes the Stratonovich differential, we see that the unique way to write $X_{s, t}$ in the form (4.1) is

$$
\begin{aligned}
X_{s, t}= & \mathbf{1}+\sum_{i=1}^{d} X_{s, t}^{i} \bullet_{i}+\sum_{i, j=1}^{d} \int_{s}^{t} X_{s, u}^{i} \circ \mathrm{d} X_{u}^{j} \bullet_{i} \star \bullet_{j} \\
& +\frac{1}{2} \sum_{1 \leq i<j \leq d}\left[X^{i}, X^{j}\right]_{s, t}\left[\bullet_{i}, \bullet_{j}\right]-\sum_{1 \leq i<j \leq d}\left[X^{i}, X^{j}\right]_{s, t}\left[\bullet_{i}\right]_{j} \\
& -\frac{1}{2} \sum_{i=1}^{d}\left[X^{i}, X^{i}\right]_{s, t}\left[\bullet_{i}\right]_{i},
\end{aligned}
$$

where $\left[\bullet_{i}, \bullet_{j}\right]=\bullet_{i} \star \bullet_{j}-\bullet_{j} \star \bullet_{i}$ is the usual Lie bracket in $\mathcal{H}^{2}$.

It follows that the corresponding geometric $\Pi$-rough path (or simply $p$-rough path if we ignore the refined $\Pi$-variation) over $B_{k}$ is given, in the first $d$ components, by the canonical (Stratonovich) geometric lift of $\left(X^{i}\right)_{i=1}^{d}$ with a bounded variation drift added to the Lévy area, and, in the remaining $\frac{d(d+1)}{2}$ components, by another bounded variation drift.

Let us now specialise to the case that $\left(X^{i}\right)_{i=1}^{d}$ is a Brownian motion with zero mean and covariance $\left[X^{i}, X^{j}\right]_{s, t}=\Sigma^{i, j}(t-s)$. We can treat $X$ as a $G^{2}\left(B_{k}\right)$-valued continuous Lévy process and apply [FS17, Thm. 53] to obtain the formula

$$
\begin{aligned}
\operatorname{ESig}\left(S(X)_{0, t}\right)=\exp _{\star}\left[t \left(\frac{1}{2} \sum_{i, j=1}^{d} \Sigma^{i, j} \bullet_{i}\right.\right. & \star \bullet_{i} \\
& +\frac{1}{2} \sum_{1 \leq i<j \leq d} \Sigma^{i, j}\left[\bullet_{i}, \bullet_{j}\right] \\
& \left.\left.-\sum_{1 \leq i<j \leq d} \Sigma^{i, j}\left[\bullet_{i}\right]_{j}-\frac{1}{2} \sum_{i=1}^{d} \Sigma^{i, i}\left[\bullet_{i}\right]_{i}\right)\right],
\end{aligned}
$$

from which it is manifest that $\operatorname{ESig}\left(S(X)_{0, t}\right)$ satisfies (5.4). We conclude by Proposition 5.16 that $S(X)_{0, t}$ is the unique $\mathcal{G}$-valued random variable with the above expected signature.

\footnotetext{
${ }^{4}$ In practice, one computes $\tau \star \sigma$ by the definition of $\star$ as the dual of the Connes-Kreimer coproduct which in turn is defined in terms of admissible cuts.
} 
Remark 6.1. As pointed out in Remark 5.17 an alternative method to check the bound (5.4) is to note that we have sufficient bounds on the local $p$-variation of $X$ (as a random geometric rough path). The advantage of this method is that it readily generalises to stochastic processes for which an explicit form of the expected signature is not readily available, including a wide class of Gaussian and Markovian rough paths CLL13, CO17, FGGR16.

Remark 6.2. A series of papers BCE18, CEFMW14, EFMPW15 have studied the relation between Itô and Stratonovich iterated integrals, particularity in relation with the quasi-shuffle algebra and Hoffman's exponential. One of the main results of [BCE18] is the existence of a unique a Hopf algebra morphism $\Psi^{*}: \mathcal{H}^{*} \rightarrow \mathcal{H}^{*}$ whose adjoint is the arborification of the Hoffman exponential, see [BCE18, Thm. 2]. In particular, a level- $N$ branched rough path satisfying the shuffle identity can be mapped through $\Psi^{*}$ to a level- $N$ branched rough path satisfying the quasi-shuffle identity (e.g., Stratonovich- resp. Itô-lift of Brownian motion), which provides in this case a higher order analogue of (6.1). Note that the results of this article are somewhat distinct from those of [BCE18] since we are not directly interested in mapping one branched rough path to another, but rather in reinterpreting every branched rough path as a geometric rough path (over a different space).

\section{Appendix A. Symbolic index}

In this appendix, we collect the most used symbols of the article, together with their meaning and the page where they were first introduced.

\begin{tabular}{|c|c|c|}
\hline Symbol & Meaning & Page \\
\hline$|\sigma|$ & Number of nodes in a forest $\sigma \in \mathcal{F}$ & 4 \\
\hline $\mathcal{A}$ & Algebra morphisms $M: \hat{\mathcal{H}} \rightarrow \mathbf{L}\left(H_{M}\right)$ with $M(B) \subset \mathfrak{u}\left(H_{M}\right)$ & 15 \\
\hline $\mathcal{A}_{k}$ & $\left\{\left(r_{1}, \ldots, r_{m}\right): r_{i} \in\{1, \ldots, k\}, m \geq 0\right\}$ & 6 \\
\hline $\mathcal{A}_{s}^{\Pi}$ & $\left\{R \in \mathcal{A}_{k}: \operatorname{deg}_{\Pi}(R) \leq s\right\}$ & 6 \\
\hline$B$ & Vector subspace of $\mathcal{B}$ such that $\mathcal{H} \simeq T(B)$ as Hopf algebras & 5 \\
\hline $\mathcal{B}$ & Span of $\mathcal{T}$ & 4 \\
\hline$B_{k}$ & Subspace of $B$ spanned by its first $k$ basis elements & 8 \\
\hline $\mathcal{C}$ & The set of matrix coefficients of elements in $\mathcal{A}$ & 15 \\
\hline $\operatorname{deg}_{\Pi}(R)$ & $\sum_{j=1}^{k} \frac{n_{j}(R)}{p_{j}}$ if $\Pi=\left(p_{1}, \ldots, p_{k}\right)$ & 6 \\
\hline$d_{p \text {-var }}$ & Homogeneous $p$-variation metric & 7 \\
\hline$\delta$ & Coproduct on the Grossman-Larson algebra & 4 \\
\hline$\Delta$ & Coproduct on the Hopf algebra $T((V))$ & 4 \\
\hline$E_{a}(B)$ & $T(B)$ equipped with a universal locally $m$-convex topology & 13 \\
\hline$E(B)$ & Completion of $E_{a}(B)$ & 13 \\
\hline$G$ & Group-like elements in $E(B)$ & 13 \\
\hline $\mathcal{G}^{*}$ & Group-like elements in $\mathcal{H}^{*}$ & 4 \\
\hline $\mathcal{G}^{N}$ & Image of $\mathcal{G}^{*}$ under $\rho^{N}$ & 5 \\
\hline $\mathcal{G}$ & $\mathcal{G}^{*} \cap \hat{\mathcal{H}}$ & 14 \\
\hline $\mathcal{G}_{k}$ & $\mathcal{G} \cap P\left(B_{k}\right)$ & 14 \\
\hline$\left(\gamma_{k}\right)_{k \geq 0}$ & Sequence of semi-norms on $B$ & 13 \\
\hline$G^{(\Pi, 1)}(V)$ & Exponential of Lie algebra in $T^{(\Pi, 1)}(V)$ generated by $V$ & 7 \\
\hline
\end{tabular}




\begin{tabular}{|c|c|c|}
\hline Symbol & Meaning & Page \\
\hline $\mathcal{F}$ & Set of all forests & 4 \\
\hline $\mathcal{H}$ & Span of $\mathcal{F}$ equipped with Grossman-Larson Hopf algebra & 4 \\
\hline $\mathcal{H}^{*}$ & Space of formal series in forests & 4 \\
\hline $\mathcal{H}^{N}$ & Subspace of $\mathcal{H}^{*}$ spanned by forests with at most $N$ & 4 \\
\hline $\mathcal{H}^{(N)}$ & Subspace of $\mathcal{H}^{*}$ spanned by forests with exactly $N$ nodes & 5 \\
\hline$\hat{\mathcal{H}}$ & Completion of $\mathcal{H}$ under topology induced by $\mathcal{H} \simeq E_{a}(B)$ & 14 \\
\hline$N_{\tau}$ & Node set of a rooted tree $\tau$ & 4 \\
\hline$n_{j}(R)$ & $\left|\left\{i: r_{i}=j\right\}\right|$ if $R=\left(r_{1}, \ldots, r_{m}\right) \in \mathcal{A}_{k}$ & 6 \\
\hline$\|\cdot\|_{p \text {-var }}$ & The $p$-variation norm & 7 \\
\hline$\omega$ & A control & 5 \\
\hline$\pi^{N}$ & Projection of $\mathcal{H}^{*}$ onto $\mathcal{H}^{(N)}$ & 5 \\
\hline$\pi_{R}$ & Projection of $T((V))$ onto $V^{\otimes R}$ & 6 \\
\hline$P(V)$ & $\prod_{m=0}^{\infty} V^{\hat{\otimes} m}$ & 4 \\
\hline$\Psi$ & Algebra isomorphism from $\mathcal{H}^{\lfloor p\rfloor}$ to $T^{(\Pi, 1)}\left(B_{k}\right)$ & 9 \\
\hline$\rho^{N}$ & Projection of $\mathcal{H}^{*}$ onto $\mathcal{H}^{N}$ & 4 \\
\hline$\rho_{\Pi-v a r}$ & The $\Pi$-variation metric & 7 \\
\hline$\curvearrowright$ & A pre-Lie product $\mathcal{B} \times \mathcal{B} \rightarrow \mathcal{B}$ & 11 \\
\hline * & Product in the Grossman-Larson algebra & 4 \\
\hline$\triangle$ & $\{(s, t): 0 \leq s \leq t \leq 1\}$ & 5 \\
\hline$S(X)$ & Signature of a rough path $X$ & 5 \\
\hline $\mathcal{T}$ & Set of all rooted trees with node label set $\{1, \ldots, d\}$ & 4 \\
\hline$T((V))$ & $\prod_{m=0}^{\infty} V^{\otimes m}$, i.e., formal series of tensors of $V$ & 4 \\
\hline$T(V)$ & Finite series in $T((V))$ & 4 \\
\hline$T^{(\Pi, s)}(V)$ & Inhomogeneous tensors with $\Pi$-degree at most $s$ & 6 \\
\hline$\dot{\theta}$ & Tensor product on $T((V))$ & 4 \\
\hline$\otimes$ & Algebraic tensor product & 4 \\
\hline$\hat{\otimes}$ & Completion of the algebraic tensor product & 4 \\
\hline $\mathfrak{u}(H)$ & Anti-Hermitian operators on $H$ & 15 \\
\hline$V^{\otimes R}$ & $V^{r_{1}} \otimes \ldots \otimes V^{r_{m}}$ if $R=\left(r_{1}, \ldots, r_{m}\right) \in \mathcal{A}_{k}$ & 6 \\
\hline $\bar{X}$ & Image under $\Psi$ of a branched rough path $X$ & 9 \\
\hline
\end{tabular}

\section{REFERENCES}

[AcG80] A. A. Agra Cev and R. V. Gamkrelidze. Chronological algebras and nonstationary vector fields. In Problems in geometry, Vol. 11 (Russian), 135-176, 243. Akad. Nauk SSSR, Vsesoyuz. Inst. Nauchn. i Tekhn. Informatsii, Moscow, 1980.

[Aze82] R. Azencott. Formule de Taylor stochastique et développement asymptotique d'intégrales de Feynman. In Seminar on Probability, XVI, Supplement, vol. 921 of Lecture Notes in Math., 237-285. Springer, Berlin-New York, 1982.

[BA89] G. Ben Arous. Flots et séries de Taylor stochastiques. Probab. Theory Related Fields 81, no. 1, (1989), 29-77.

[BCCH17] Y. Bruned, A. Chandra, I. Chevyrev, and M. Hairer. Renormalising SPDEs in regularity structures. ArXiv e-prints (2017). arXiv:1711.10239

[BCE18] Y. Bruned, C. Curry, and K. Ebrahimi-Fard. Quasi-shuffle algebras and renormalisation of rough differential equations. ArXiv e-prints (2018).arXiv:1801.02964.

[BCFP17] Y. Bruned, I. Chevyrev, P. K. Friz, and R. Preiss. A Rough Path Perspective on Renormalization. ArXiv e-prints (2017). arXiv:1701.01152 
$\left[\mathrm{BFG}^{+} 17\right]$ C. Bayer, P. K. Friz, P. Gassiat, J. Martin, and B. Stemper. A regularity structure for rough volatility. ArXiv e-prints (2017). arXiv:1710.07481.

[BGly16] H. Boedihardjo, X. Geng, T. Lyons, and D. Yang. The signature of a rough path: uniqueness. Adv. Math. 293, (2016), 720-737. doi:10.1016/j.aim.2016.02.011.

[BHZ16] Y. Bruned, M. Hairer, and L. Zambotti. Algebraic renormalisation of regularity structures. ArXiv e-prints (2016). arXiv:1610.08468

[Boe15] H. Boedihardjo. Decay Rate of Iterated Integrals of Branched Rough Paths. ArXiv e-prints (2015). To appear in Ann. Inst. H. Poincaré Anal. Non Linéaire. arXiv:1501.05641 doi:10.1016/j.anihpc.2017.09.002

[But16] J. C. Butcher. Numerical methods for ordinary differential equations. John Wiley \& Sons, Ltd., Chichester, third ed., 2016, xxiii+513. With a foreword by J. M. SanzSerna.

[CeFMW14] C. Curry, K. Ebrahimi-Fard, S. J. A. Malham, and A. Wiese. Lévy processes and quasi-shuffle algebras. Stochastics 86, no. 4, (2014), 632-642. doi:10.1080/17442508.2013.865131

[Cha10] F. Снаротоn. Free pre-Lie algebras are free as Lie algebras. Canad. Math. Bull. 53, no. 3, (2010), 425-437. doi:10.4153/CMB-2010-063-2

[Che57] K.-T. Chen. Integration of paths, geometric invariants and a generalized BakerHausdorff formula. Ann. of Math. (2) 65, (1957), 163-178.

[Che18] I. Chevyrev. Random walks and Lévy processes as rough paths. Probab. Theory Related Fields 170, no. 3-4, (2018), 891-932. doi:10.1007/s00440-017-0781-1

[CL01] F. Снаротоn and M. Livernet. Pre-Lie algebras and the rooted trees operad. Internat. Math. Res. Notices, no. 8, (2001), 395-408. doi:10.1155/S1073792801000198.

[CL16] I. Chevyrev and T. Lyons. Characteristic functions of measures on geometric rough paths. Ann. Probab. 44, no. 6, (2016), 4049-4082. doi:10.1214/15-A0P1068

[CLL13] T. Cass, C. Litterer, and T. Lyons. Integrability and tail estimates for Gaussian rough differential equations. Ann. Probab. 41, no. 4, (2013), 3026-3050.

[CO17] T. Cass and M. Ogrodnik. Tail estimates for Markovian rough paths. Ann. Probab. 45, no. 4, (2017), 2477-2504.

[CW16] T. CASS and M. P. WEIDNER. Tree algebras over topological vector spaces in rough path theory. ArXiv e-prints (2016). arXiv:1604.07352

[EFMPW15] K. Ebrahimi-Fard, S. J. A. Malham, F. Patras, and A. Wiese. The exponential Lie series for continuous semimartingales. Proc. A. 471, no. 2184, (2015), 20150429, 19. doi:10.1098/rspa.2015.0429.

[FGGR16] P. K. Friz, B. Gess, A. Gulisashvili, and S. Riedel. The Jain-Monrad criterion for rough paths and applications to random Fourier series and non-Markovian Hörmander theory. Ann. Probab. 44, no. 1, (2016), 684-738. doi:10.1214/14-A0P986.

[Foi02] L. Foissy. Finite-dimensional comodules over the Hopf algebra of rooted trees. $J$. Algebra 255, no. 1, (2002), 89-120. doi:10.1016/S0021-8693(02)00110-2.

[FS82] G. B. Folland and E. M. Stein. Hardy spaces on homogeneous groups, vol. 28 of Mathematical Notes. Princeton University Press, Princeton, N.J.; University of Tokyo Press, Tokyo, 1982, xii +285 .

[FS17] P. Friz and A. Shekhar. General rough integration, Lévy rough paths and a Lévy-Kintchine type formula. Ann. Probab. 45, no. 4, (2017), 2707-2765. doi:10.1214/16-AOP1123.

[FV08] P. Friz and N. Victoir. Euler estimates for rough differential equations. J. Differential Equations 244, no. 2, (2008), 388-412.

[FV10] P. K. Friz and N. B. Victoir. Multidimensional stochastic processes as rough paths, vol. 120 of Cambridge Studies in Advanced Mathematics. Cambridge University Press, Cambridge, 2010, xiv +656 .

[Gub10] M. Gubinelli. Ramification of rough paths. J. Differential Equations 248, no. 4, (2010), 693-721. doi:10.1016/j.jde.2009.11.015

[GV95] A. Giambruno and A. Valenti. On minimal *-identities of matrices. Linear and Multilinear Algebra 39, no. 4, (1995), 309-323. doi:10.1080/03081089508818405.

[Gyu08] L. G. GyurKó. Numerical methods for approximating solutions to rough differential equations. Ph.D. thesis, University of Oxford, 2008. 
[Gyu16] L. G. Gyurkó. Differential equations driven by П-rough paths. Proc. Edinb. Math. Soc. (2) 59, no. 3, (2016), 741-758.

[Hai14] M. Hairer. A theory of regularity structures. Invent. Math. 198, no. 2, (2014), 269-504. doi:10.1007/s00222-014-0505-4.

[HK15] M. Hairer and D. Kelly. Geometric versus non-geometric rough paths. Ann. Inst. Henri Poincaré Probab. Stat. 51, no. 1, (2015), 207-251. doi:10.1214/13-AIHP564.

[HL10] B. HAmBLY and T. Lyons. Uniqueness for the signature of a path of bounded variation and the reduced path group. Ann. of Math. (2) 171, no. 1, (2010), 109167. doi:10.4007/annals.2010.171.109

[HS90] W. Hebisch and A. Sikora. A smooth subadditive homogeneous norm on a homogeneous group. Studia Math. 96, no. 3, (1990), 231-236.

[LV04] T. Lyons and N. Victoir. Cubature on Wiener space. Proc. R. Soc. Lond. Ser. A Math. Phys. Eng. Sci. 460, no. 2041, (2004), 169-198. Stochastic analysis with applications to mathematical finance. doi:10.1098/rspa.2003.1239

[LV06] A. Lejay and N. Victoir. On $(p, q)$-rough paths. J. Differential Equations 225, no. 1, (2006), 103-133.

[LV07] T. Lyons and N. Victoir. An extension theorem to rough paths. Ann. Inst. H. Poincaré Anal. Non Linéaire 24, no. 5, (2007), 835-847.

[Lyo98] T. J. Lyons. Differential equations driven by rough signals. Rev. Mat. Iberoamericana 14, no. 2, (1998), 215-310. doi:10.4171/RMI/240

H. Boedihardjo, Department of Mathematics and Statistics, University of Reading, Reading RG6 6AX, United Kingdom

E-mail address: h.s.boedihardjo@reading.ac.uk

I. Chevyrev, Mathematical Institute, University of Oxford, Andrew Wiles Building, Radcliffe Observatory Quarter, Woodstock Road, Oxford OX2 6GG, United KINGDOM

E-mail address: chevyrev@maths.ox.ac.uk 